\title{
Deep MAGIC observations of the Galactic Centre region
}

\section{Fruck*t}

Physics Dept., Technical University Munich, Germany

E-mail: cfruck@ph.tum.de

\section{Vovk, M. Strzys}

Max-Planck-Institut für Physik, Munich, Germany

E-mail: ievgen.vovk@mpp.mpg.de, strzys@mpp.mpg.de

\section{Y. Iwamura}

ICRR, Tokyo, Japan

E-mail: iwamuraeicrr.u-tokyo.ac.jp

\section{S. Ventura}

University of Siena \& INFN Pisa, Italy

E-mail: sofia.venturaepi.infn.it

\section{for the MAGIC Collaboration $\ddagger$}

\begin{abstract}
Dense molecular clouds in the Central Molecular Zone (CMZ) filling the $\sim 200 \mathrm{pc}$ region around the Galactic Center (GC) allow to use gamma rays as tracers for cosmic rays (CRs). We present the updated analysis covering more than 100 hours of MAGIC observations of this region, which is believed to host a PeV proton accelerator. We use new analysis tools that were specially developed for such a task for computing the instrument responses, studying the morphology and extracting energy spectra of the extended gamma-ray emission. We observe no significant difference between the diffuse emission spectra of the immediate Galactic Center vicinity and the rest of the Galactic Ridge. Though the derived best fit CR profile is compatible with the $1 / \mathrm{r}$ radial distance scaling, we argue that this measurement is presently limited by the our knowledge of the gas distribution in $3 \mathrm{D}$ at the GC.
\end{abstract}

36th International Cosmic Ray Conference -ICRC2019-

July 24th - August 1st, 2019

Madison, WI, U.S.A.

\footnotetext{
* Speaker.

${ }^{\dagger}$ Formerly at Max-Planck-Institut für Physik, Munich, Germany

${ }_{\ddagger}^{\ddagger}$ https: / /magic.mpp.mpg. de/. For collaboration list see PoS(ICRC2019)1177
} 


\section{Introduction}

The Galactic Centre (GC) is one of the densest and astrophysically 'richest' regions in our galaxy. It hosts a super-massive black hole with $4 \times 10^{6} M_{\odot}$, associated with the point-like radio source SgrA*, which is remarkably dim in radio and X-ray [11]. A TeV gamma-ray source at the Galactic Centre can probably also be associated with $\operatorname{SgrA} *[7,4,1]$. The mechanism by which this object produces those $\mathrm{TeV}$ photons gives rise to speculations, but still largely remains unknown $[5,14,8]$. Apart from the point-like gamma-ray source at the coordinates of $\mathrm{SgrA}^{*}$, extended emission stretching around 2 degrees along the Galactic plane and tracing the distribution of molecular gas has been found by all three major Cherenkov telescope installations $[6,3,1]$. In this case, a hadronic origin of the gamma rays seems most likely as the gamma-ray intensity traces the density of molecular clouds in that region and thus favors the idea of highly energetic protons interacting and producing pions. More recent studies have found evidence that the source of those high energy protons could indeed be the location of SgrA* and therefore possibly linked to the super-massive black hole. This conclusion is founded on two observations: a) the comparably hard spectral shape (index $\approx 2$ ) that extends well beyond $10 \mathrm{TeV}$ in energy, $b$ ) the radial profile of the underlying cosmic-ray $(\mathrm{CR})$ density that is needed to explain the gamma-ray emission given the underlying gas density of the molecular clouds [13].

\section{The MAGIC observation campaign and data analysis}

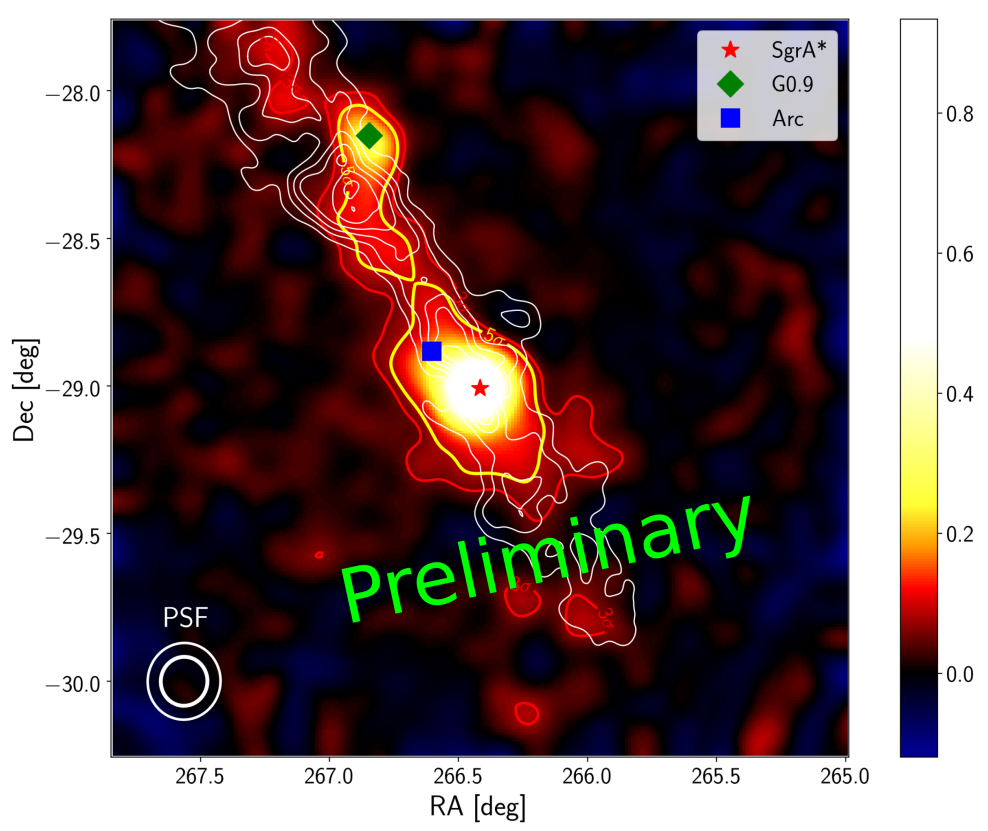

Figure 1: Sky map of the $2.5 \times 2.5$ degrees around the GC. The three point-like gamma-ray sources $(\operatorname{SgrA} *$, G09, Arc) are highlighted with markers and the gas density traced by CS radio emission is shown by white contours [15]. The red and yellow contours highlight regions corresponding to more than 3 and 5 sigma deviations from the background test statistic, respectively. 


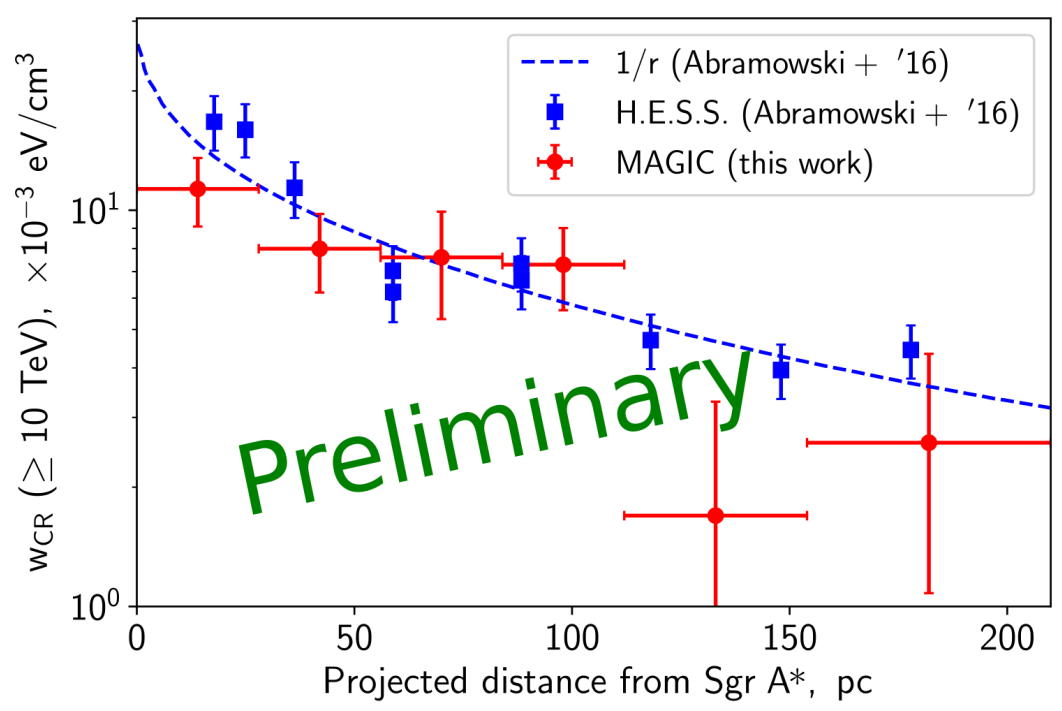

Figure 2: MAGIC CR density profile as a function of distance from the GC, compared to the H.E.S.S. measurement. This measurement has been obtained using events with $E>1 \mathrm{TeV}$ and applying the method described in [13].

MAGIC has observed the GC region for more than $100 \mathrm{~h}$ between April 2012 and May 2017, in the season from March to July. The observations were carried out in the so-called "Wobble" mode [9] with a pointing offset of $0.4^{\circ}$, centred on $\mathrm{Sgr} \mathrm{A}^{*}$. Due to the low culmination $(\approx 58 \mathrm{deg}$ when observed from La Palma), all observations were conducted at large zenith distances (58 deg - $70 \mathrm{deg}$ ), resulting in a higher energy threshold, but also a larger effective collection area (see [1] for details). The data have been processed with the standard MAGIC Analysis and Reconstruction Software (MARS [17]). This procedure includes the computation of image and stereo parameters and the exclusion of bad data quality periods such as low atmospheric transmission or a too high level of background light. The machine learning technique Random Forests, trained on image parameters of $\mathrm{MC}$ and real data is used for the direction reconstruction, energy estimation and background reduction [2]. The high-level analysis has been preformed using the SkyPrism [16] package, which includes tools for computing energy-binned sky maps and instrument response functions, as well as a tool-set for performing spatial and cross-bin (simultaneous fit to energy binned sky maps across energy bins, using spectral model for each spatial component) likelihood fits on the data.

A sky map of the $2.5 \times 2.5$ degrees region around the GC is shown in Figure 1. Apart from the three point sources (SgrA*, G09, Radio Arc) extended gamma-ray emission has been found in regions with high density of molecular gas, as traced by CS radio emission [15]. Assuming that the density and distribution of target material is known, the energy density of CRs can be reconstructed out of the gamma-ray luminosity. Figure 2 shows the derived CR density as a function of distance from the GC, applying a similar method like the one described by [13]. The gamma-ray flux has been extracted by cutting the CS map into concentric rings and fitting them simultaneously, together with the three point-like sources to the gamma-ray sky map above $1 \mathrm{TeV}$ in energy. This 


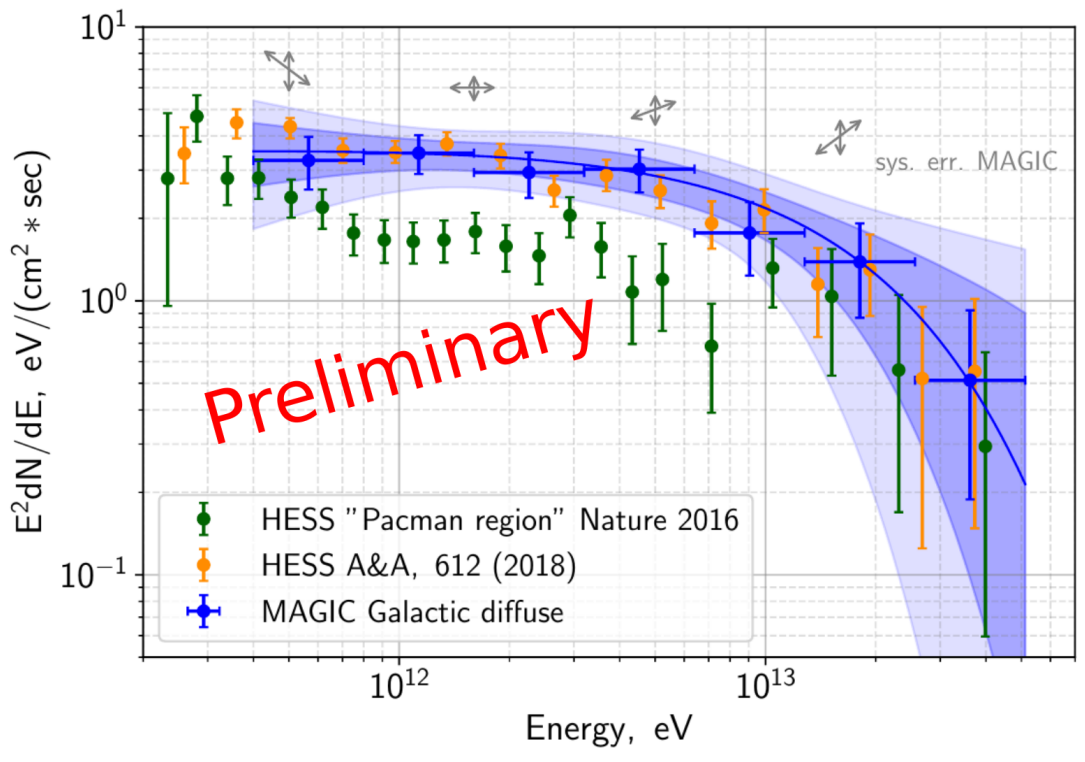

Figure 3: SEDs of the Diffuse Component, compared to the two published spectra measured by H.E.S.S., in the inner region around SgrA* (green, [13]) and from a more extended region along the Galactic plane that roughly corresponds to he region probed in this analysis (orange, [12]).

measurement is compatible with the assumption of a $1 / \mathrm{r}$ profile of the $\mathrm{CR}$ density, which would favor diffusion from a central source, which could be SgrA*. In order to study the spectral shape of the diffuse gamma rays emitted along the central molecular zone (CMZ), the data has been split into 7 bins in energy, equally spaced on a logarithmic axis and ranging from $400 \mathrm{GeV}$ to $50 \mathrm{TeV}$. The energy spectrum has been derived in two different ways: bin-wise spatial likelihood fit, and using a cross-bin spectral likelihood fit. For both methods a single spatial model has been fitted to the energy-binned sky map. The model consists of 3 point sources (SgrA*, G09, Arc), the model for the diffuse emission (CS map scaled with the 1/r CR profile), and a model for the irreducible background that has been generated from OFF-source regions using SkyPrism. The resulting SED of the diffuse component is shown in Figure 3. The bin-wise SED has been obtained by fitting the multi-component model to each bin individually. A spillover-correction has been applied, based on the energy migration matrix that has been computed from MC events. The solid line and the filled bands were computed by fitting a spectral model of the general form

$$
d N / d E=N\left(\frac{E}{E_{0}}\right)^{\Gamma} \exp \left(-E / E_{c u t}\right)
$$

(power law for G0.9 and Arc, power law with ct-off for SgrA* and Diffuse) to all energy bins simultaneously, using the forward-folding technique. During this process, flux maps are first computed in bins of true energy, and then folded with the instrument response functions (IRFs) that include the energy migration matrix. In order to improve the quality of the error calculation, MCMC sampling has been applied, using the emcee Python package [10]. The best-fit parameters for the diffuse component are $N=9.32_{-1.63}^{+2.39} \times 10^{-25} \mathrm{eV}^{-1} \mathrm{~cm}^{-2} \mathrm{~s}^{-1}, \Gamma=-1.98_{-0.21}^{+0.26}$ and $E_{\text {cut }}=17.5_{-9.55}^{+59.3} \mathrm{TeV}$. 


\section{Discussion}

The results of the MAGIC observation campaign of the GC are largely consistent with measurements by H.E.S.S. [13, 12] (see Figure 3). The spectrum observed by [13] has been measured in a region that is much closer to SgrA*, compared to [12] and the data presented here. Nevertheless, the overall spectral shape is compatible, even if there is a hint for a turn-over in the MAGIC spectrum above around $10 \mathrm{TeV}$. The power law with cut-off as spectral model is preferred over a single power law by $\approx 2 \sigma$. A power-law extension of the gamma-ray spectrum beyond $100 \mathrm{TeV}$ is still possible within a statistical error margin of $2 \sigma$. Therefore the PeVatron scenario cannot be ruled out, even if the data show evidence that the gamma-ray spectrum may cut-off before reaching $100 \mathrm{TeV}$. Our data also confirm the apparent radial profile in the CR density that seems to peak towards the GC. This supports the idea of CRs being produced at the GC and diffusing outwards, thereby "illuminating" the molecular clouds on their way. These CRs could also have been produced during a past episode of increased activity of the central $\mathrm{BH}$, as observations of the current brightness of SgrA* in radio and X-ray do not support the scenario of a large enough quantity of protons being accelerated to $\mathrm{TeV}$ energies at present times. The biggest uncertainty when trying to reconstruct the CR density profile is introduced by a lack of knowledge about the actual 3D distribution of the gas around the GC. The closer one approaches the GC and therefore the center of rotational motion, the less accurate the estimation of the Z-coordinate becomes. The actual distance of molecular clouds to the GC can therefore not be determined accurately, leading to systematic errors, especially when approaching the GC closer than about $20 \mathrm{pc}$ in projected distance. In the future even deeper and better resolved observations with CTA might allow for a better understanding of the distribution, spectrum and origin of the CRs in the vicinity of the GC, if the gamma-ray observations are also matched with a more precise model of the 3D distribution of the dense gas in the region.

\section{Acknowledgments}

We would like to thank the Instituto de Astrofísica de Canarias for the excellent working conditions at the Observatorio del Roque de los Muchachos in La Palma. The financial support of the German BMBF and MPG, the Italian INFN and INAF, the Swiss National Fund SNF, the ERDF under the Spanish MINECO (FPA2015-69818-P, FPA2012-36668, FPA2015-68378P, FPA2015-69210-C6-2-R, FPA2015-69210-C6-4-R, FPA2015-69210-C6-6-R, AYA2015-71042P, AYA2016-76012-C3-1-P, ESP2015-71662-C2-2-P, FPA2017-90566-REDC), the Indian Department of Atomic Energy, the Japanese JSPS and MEXT, the Bulgarian Ministry of Education and Science, National RI Roadmap Project DO1-153/28.08.2018 and the Academy of Finland grant nr. 320045 is gratefully acknowledged. This work was also supported by the Spanish Centro de Excelencia "Severo Ochoa" SEV-2016-0588 and SEV-2015-0548, and Unidad de Excelencia "María de Maeztu" MDM-2014-0369, by the Croatian Science Foundation (HrZZ) Project IP2016-06-9782 and the University of Rijeka Project 13.12.1.3.02, by the DFG Collaborative Research Centers SFB823/C4 and SFB876/C3, the Polish National Research Centre grant UMO2016/22/M/ST9/00382 and by the Brazilian MCTIC, CNPq and FAPERJ. 


\section{References}

[1] M. L. Ahnen, S. Ansoldi, L. A. Antonelli, et al. Observations of Sagittarius A* during the pericenter passage of the G2 object with MAGIC. A\&A, 601:A33, May 2017.

[2] Albert et al. Implementation of the Random Forest method for the Imaging Atmospheric Cherenkov Telescope MAGIC. Nuclear Instruments and Methods in Physics Research A, 588:424-432, April 2008.

[3] A. Archer et al. TeV Gamma-ray Observations of The Galactic Center Ridge By VERITAS. ArXiv e-prints: 1602.08522, February 2016.

[4] D. R. Ballantyne, Fulvio Melia, Siming Liu, and Roland M. Crocker. A Possible Link between the Galactic Center HESS Source and Sagittarius A*. The Astrophysical Journal Letters, 657(1):L13, February 2007.

[5] M. Chernyakova, D. Malyshev, F. A. Aharonian, R. M. Crocker, and D. I. Jones. THE HIGHENERGY, ARCMINUTE-SCALE GALACTIC CENTER GAMMA-RAY SOURCE. The Astrophysical Journal, 726(2):60, January 2011.

[6] The H. E. S. S. Collaboration and F. A. Aharonian. Discovery of Very-High-Energy GammaRays from the Galactic Centre Ridge. Nature, 439(7077):695-698, February 2006. arXiv: astro-ph/0603021.

[7] The HESS Collaboration and F. Acero. Localising the VHE gamma-ray source at the Galactic Centre. Monthly Notices of the Royal Astronomical Society, 402(3):1877-1882, March 2010. arXiv: 0911.1912.

[8] M. Fatuzzo and F. Melia. DIFFUSIVE COSMIC-RAY ACCELERATION IN SAGITTARIUS Alast. The Astrophysical Journal, 757(1):L16, September 2012.

[9] V. P. Fomin, A. A. Stepanian, R. C. Lamb, D. A. Lewis, M. Punch, and T. C. Weekes. New methods of atmospheric Cherenkov imaging for gamma-ray astronomy. I. The false source method. Astroparticle Physics, 2:137-150, May 1994.

[10] Daniel Foreman-Mackey, David W. Hogg, Dustin Lang, and Jonathan Goodman. emcee: The MCMC Hammer. Publications of the Astronomical Society of the Pacific, 125(925):306, 2013.

[11] Reinhard Genzel, Frank Eisenhauer, and Stefan Gillessen. The galactic center massive black hole and nuclear star cluster. Reviews of Modern Physics, 82(4):3121, 2010.

[12] H.E.S.S. Collaboration, :, H. Abdalla, A. Abramowski, F. Aharonian, F. Ait Benkhali, A. G. Akhperjaniany, T. Andersson, E. O. Angüner, M. Arakawa, and et al. Characterising the VHE diffuse emission in the central 200 parsecs of our Galaxy with H.E.S.S. ArXiv e-prints, June 2017. 
[13] H.E.S.S. Collaboration, A. Abramowski, F. Aharonian, F. A. Benkhali, A. G. Akhperjanian, E. O. Angüner, M. Backes, A. Balzer, Y. Becherini, J. B. Tjus, and et al. Acceleration of petaelectronvolt protons in the Galactic Centre. Nature, 531:476-479, March 2016.

[14] Tim Linden, Elizabeth Lovegrove, and Stefano Profumo. THE MORPHOLOGY OF HADRONIC EMISSION MODELS FOR THE GAMMA-RAY SOURCE AT THE GALACTIC CENTER. The Astrophysical Journal, 753(1):41, July 2012.

[15] M. Tsuboi, T. Handa, and N. Ukita. Dense Molecular Clouds in the Galactic Center Region. I. Observations and Data. Astrophysical Journal, Supplement, 120:1-39, January 1999.

[16] I. Vovk, M. Strzys, and C. Fruck. Spatial likelihood analysis for MAGIC telescope data. From instrument response modelling to spectral extraction. Astronomy and Astrophysics, 619:A7, Nov 2018.

[17] R. Zanin, E. Carmona, J. Sitarek, P. Colin, and K. Frantzen. Mars, the magic analysis and reconstruction software. In Proc. of the 33st International Cosmic Ray Conference, Rio de Janeiro, Brasil, 2013. 